The International

Journal of

Integrated

Engineering

\title{
Stair-Hexagonal Slot Antenna with Coplanar Waveguide Technique for Biomedical Applications
}

\author{
Yusnita Rahayu ${ }^{*}$, M. Fadhlurrahman Hilmi², Yuyu Wahyu ${ }^{3}$ \\ ${ }^{1,2}$ Department of Electrical Engineering, Faculty of Engineering, Universitas Riau, \\ Kampus Bina Widya Km 12.5 Simpang Baru, Pekanbaru, 28293, INDONESIA \\ ${ }^{3}$ Electronic and Telecommunication Research Center, Indonesian Institue of Sciences (LIPI), \\ Jl. Sangkuriang - Komplek LIPI Gedung 20, Lt. 4, Bandung, 40135, INDONESIA \\ *Corresponding Author
}

DOI: https://doi.org/10.30880/ijie.2020.12.06.021

Received 31 March 2020; Accepted 19 July 2020; Available online 30 July 2020

\begin{abstract}
Breast cancer is the most common disease suffered by women and increases significantly. UltraWideband (UWB) is a technology that applies electromagnetic signals with wide bandwidth that are currently widely used in the medical field. This research introduces the design of the Stair-Hexagonal slot antenna with the Coplanar Waveguide (CPW) technique which operated at desired frequency of $5.8 \mathrm{GHz}$. Firstly, the design process of the antenna is adding stair slot to get wide bandwidth. Secondly by adding Hexagonal slot to get the best $S_{11}$ parameter at the desired frequency. The purpose of antenna is to detect an object in body organs such as tumors and cancer. The antenna is printed and tested. The measured return loss shows that the antenna works well in the desired frequency with wider bandwidth than simulated one. The measured bandwidth of $7.88 \mathrm{GHz}$ is achieved. The gain of the antenna from the simulation is $2.42 \mathrm{dBi}$ and radiation pattern of simulation and measurement are bidirectional, wherein the highest gain is in the rear position. Further analysis is done by comparing the proposed antenna with the existing related works. The result of this research shows the practical usage of the flexible substrate because it can increase the accuracy of object detection.
\end{abstract}

Keywords: Ultrawideband (UWB), Coplanar Waveguide (CPW), Flexible antenna

\section{Introduction}

Nowadays, the interest of the scientific community towards the application of ultra-wideband (UWB) for biomedical and other diagnostic purposes at microwave frequencies has become prominent [1]. Breast cancer detection using ultrawideband technique is one of the applications. It is the most common disease suffered by women and increases significantly. According to the International Agency for Research on Cancer (IARC), about 9.6 million people died from cancer in 2018, and it is estimated that cancer patients around the world will increase by 18.1 million cases [2]. However, if breast cancer can be detected early, then the five-year survival rate is over $90 \%$ [3].

Current methods for breast imaging are Ultrasound, Mammography, and Magnetic Resonance Imaging (MRI). Mammography uses low energy to be used in breast cancer diagnosis, but it has several shortcomings. Several of its shortcomings are ionizing radiation that causes a health hazard, while the emphasis on the breast causes discomfort in the patient. Further, mammography needs the breast to be pressed during the examination, which can cause pain in the patient. Moreover, tumors located close to the chest wall or underarm result in a $10 \%$ - 30\% rate of false negatives [4].

Ultrasound is a common technique used to diagnose breast tumors but has a lack of detection. It is because ultrasound used sound waves, and the reflection of sound waves will be processed into images of the breast. The accuracy of reflected waves depends on the dimensions of the body, which makes ultrasound has a high false rate. While MRI technology is 
expensive and complicated, because this is a kind of high-performance technology using the magnetic field to align hydrogen atomic protons to be resonance, it used radio frequency to trigger and produce high-resolution images [5].

Recently, many researchers have researched breast cancer detection by using a microwave imaging system [6]. One of the technologies that apply microwave imaging is UWB. The UWB was initiated by FCC (Federal Communications Commission) in 2002. The FCC defines UWB has bandwidth over $500 \mathrm{MHz}$ with a frequency range of 3.1 up to 10.6 [7]. This range frequencies allow us to have a good resolution and a sufficient penetration depth for breast imaging simultaneously. The antenna is one of the systems that applies UWB technology. Antennas are important components in wireless communication, which have advantages, including lightweight, low profile, cost-effective, efficiency, and reliability [14]. So that it can be used for biomedical applications with these advantages. By using the antenna, the transmitted signal can directly hit the object, which depending on the radiation pattern [8].

In this paper, a UWB antenna is presented for biomedical applications. The antenna uses Polymide Terephthalate (PET) substrate material with relative permittivity of 3.0. The square antenna with the stair-hexagonal slot is designed. The simulation is carried out using CST studio suite software.

\section{Methodology}

\subsection{Mathematical Equation}

There is a basic equation in the antenna design for obtaining the S11 parameter at the desired frequency. The following are formulas for determining the dimensions of the patch for rectangular shape before characterized [13]:

Width (W) calculation:

$$
W=\frac{c}{2 f_{0} \sqrt{\frac{\left(\varepsilon_{r}+1\right)}{2}}}
$$

Calculation of the Effective dielectric constant is based on parameters of the thickness, dielectric constant of the substrate, and the width of the patch antenna.

$$
\varepsilon_{e f f}=\frac{\varepsilon_{r}+1}{2}+\frac{\varepsilon_{r}-1}{2}\left[1+12 \frac{h}{W}\right]^{-\frac{1}{2}}
$$

Effective length calculation:

$$
L_{e f f}=\frac{c}{2 f_{0} \sqrt{\varepsilon_{e f f}}}
$$

Length extension $\Delta L$ calculation:

$$
\Delta L=0.412 h \frac{\left(\varepsilon_{e f f}+0.3\right)\left(\frac{W}{h}+0.264\right)}{\left(\varepsilon_{e f f}-0.258\right)\left(\frac{W}{h}+0.8\right)}
$$

The actual length of the patch calculation:

$$
L=L_{e f f}-2 \Delta L
$$

\section{Where the following parameters used are:}

$f_{0}=$ the resonance frequency

$\varepsilon_{0}=$ the relative permittivity of the dielectric substrate

$\mathrm{c}=$ the speed of light: $3 \times 10^{8}$

$\mathrm{W}=$ the width of the patch

$\mathrm{L}=$ the length of the patch

$\mathrm{h}=$ the thickness 


\subsection{Optimization and Characterization}

The initial structure of an antenna is a rectangular patch with additional stair-hexagonal slots printed on the PET substrate with relative permittivity $\varepsilon_{\mathrm{r}}=3.0$, and thickness of $0.135 \mathrm{~mm}$. The antenna is designed with a CPW technique. In order to increase the antenna performance, characterization and optimization of the structure have been performed to obtain the optimal $\mathrm{S}_{11}$ parameter at the desired frequency of $5.8 \mathrm{GHz}$. Adding the hexagonal slots on the antenna affects the operating frequency, while the stairs increase the bandwidth. The followings are the characterization of an antenna carried out in this paper.

Fig. 1 shows the comparison of $\mathrm{S}_{11}$ before and after stairs added on the patch. It shows that the stairs on the patch provide wider bandwidth than the patch without it. The bandwidth of antenna without stair slot is $2.21 \mathrm{GHz}$, while the bandwidth of antenna with stair slot is $3.11 \mathrm{GHz}$.

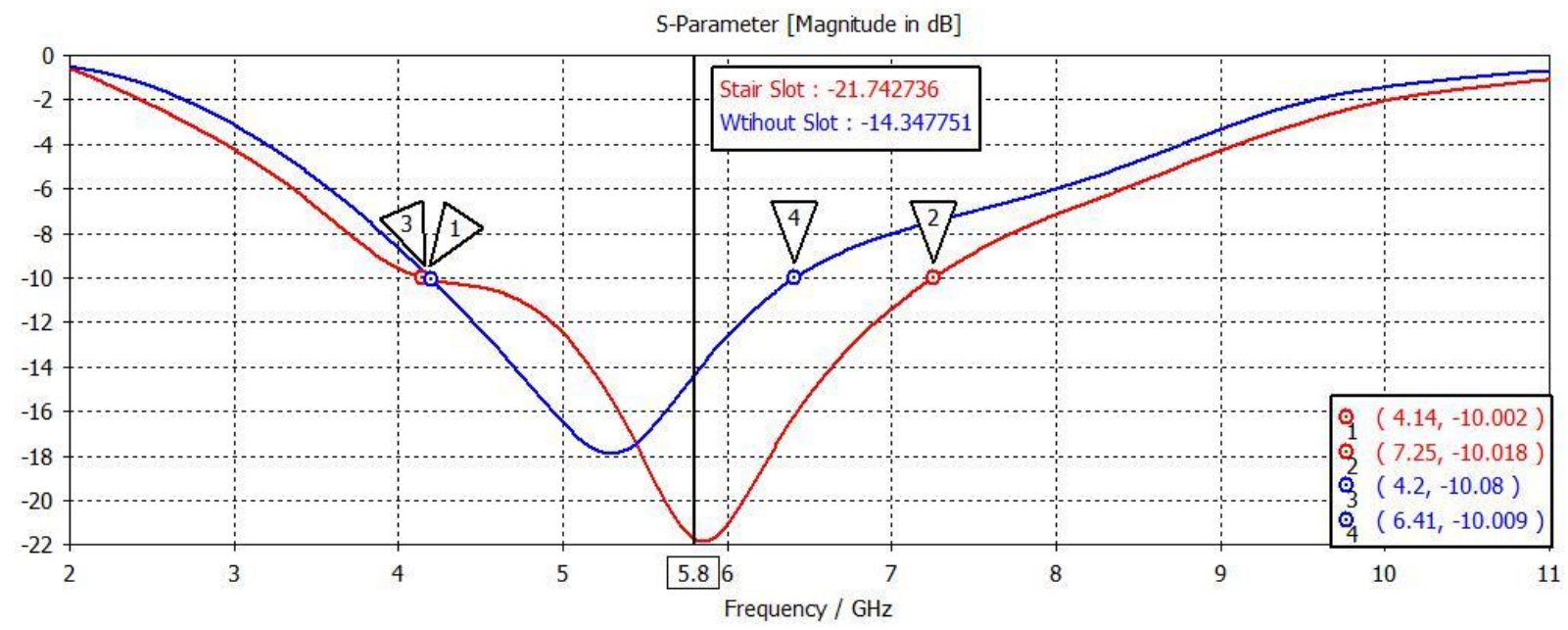

Fig. 1 - Comparison S11 before and after stairs added to the patch antenna

Table 1 shows the effect of the hexagonal slot on the frequency response. It shows increasing the number of the hexagonal slots of an antenna has shifted the frequency because the value of the s11 parameter is different with each additional slot. Fig. 2 presents the geometry of the antenna with the hexagonal slots.

Table 1 - The effect of the hexagonal slot on antenna performance

\begin{tabular}{ccc}
\hline Slot & $\begin{array}{c}\text { S11 parameter } \\
(\mathbf{d B})\end{array}$ & $\begin{array}{c}\text { Bandwidth } \\
(\mathbf{G H z})\end{array}$ \\
\hline 1 & -21.41 & 2.9 \\
2 & -21.26 & 3 \\
3 & -20.23 & 3.07 \\
4 & -22.716 & 3.1 \\
\hline
\end{tabular}

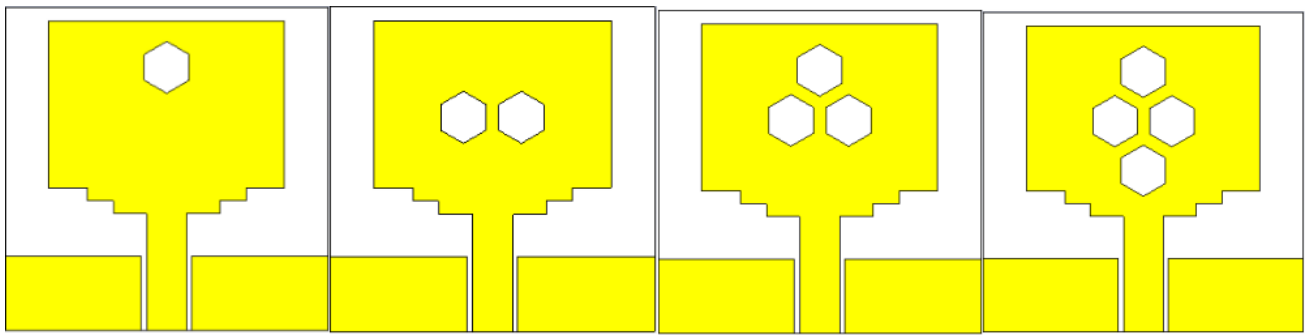

(a)

(b)

(c)

(d)

Fig. 2 - The geometry antenna with the hexagonal slot (a) 1 slot; (b) 2 slots; (c) 3 slots; (d) 4 slots 


\section{Antenna Model}

Antenna model is the final design of the antenna for fabrication, wherein the final design there are additional slots on the ground and trimming on the left and right side of the transmission line, or it is called Coplanar Waveguide (CPW). CPW technique is used because the PET substrate can only be printed on one side. The addition of slots on the ground does not significantly affect changes in simulation results while trimming the left and right sides of the transmission line aim to make the SMA connector soldering process easier because only the middle feed is connected to the transmission line.

Fig. 3 shows the proposed design of an antenna with stair-hexagonal slots operated at $5.8 \mathrm{GHz}$. The dimensions of an antenna are listed in Table 2.

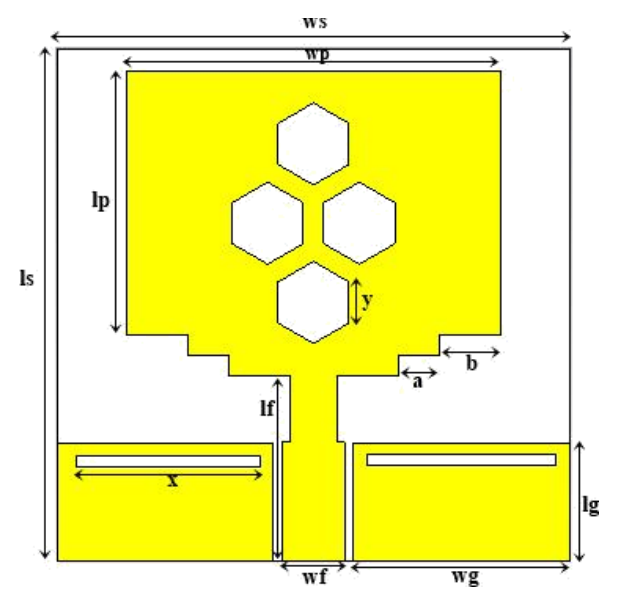

Fig. 3 - The geometry of the proposed antenna

Table 2 - Dimensions of the proposed antenna

\begin{tabular}{cc}
\hline Parameter & Dimension $(\mathbf{m m})$ \\
\hline ws & 25 \\
ls & 25 \\
wp & 18.27 \\
lp & 14.89 \\
wf & 3.1 \\
lf & 10.15 \\
wg & 10.55 \\
lg & 5.8 \\
a & 2 \\
b & 3 \\
y & 2 \\
x & 9.1 \\
\hline
\end{tabular}

Fig. 4 shows the prototype antenna printed on a PET substrate. The antenna was printed using the inkjet printer. The position of the SMA connector is perpendicular to the antenna. The process of soldering antenna with SMA connector requires the addition of another material, namely conductive glue, which aims to make the soldering process more effective so that it does not damage the substrate and is not easily detached. 


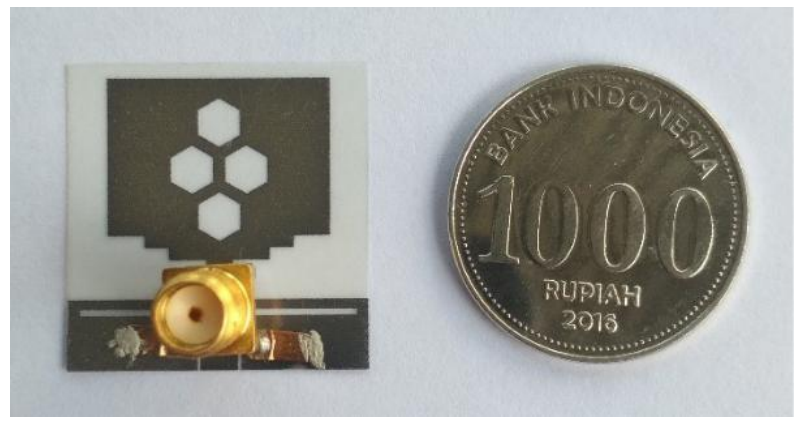

Fig. 4 - The prototype of the antenna

\section{Results and Discussions}

A comparison between both simulated and measured return loss will be discussed in this section. The antenna should meet the minimum UWB bandwidth requirement in the frequency range. Fig. 5 shows the comparison for both simulated and measured $\mathrm{S}_{11}$ parameters of the antenna. The simulated result shows that the antenna works well in the range of 4.12-7.375 GHz with respect to $-10 \mathrm{~dB}$. It reaches $3.255 \mathrm{GHz}$ of bandwidth with a return loss of $-23.075 \mathrm{~dB}$ at $5.8 \mathrm{GHz}$. However, the prototype antenna works in the range of $2.08-9.96 \mathrm{GHz}$ with respect to $-10 \mathrm{~dB}$. The measured result provides $7.88 \mathrm{GHz}$ of bandwidth with a return loss of $-22.5 \mathrm{~dB}$ at $5.8 \mathrm{GHz}$. The difference in results between simulation and measurement is due to a lack of information regarding the specifications of the PET substrate made at CST Studio. Therefore there is a slight difference in results between simulation and measurement. The minimum bandwidth of the UWB requirement was achieved.

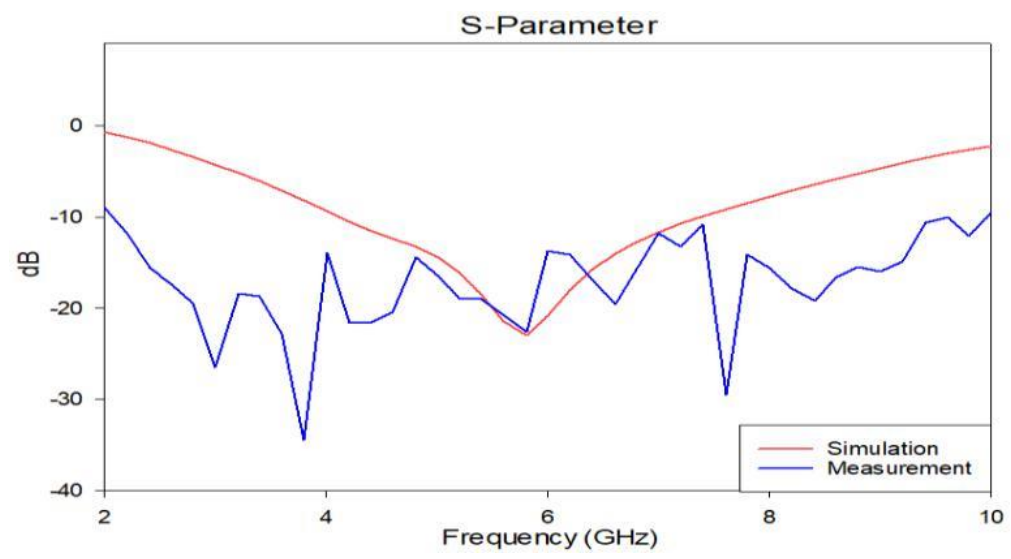

Fig. 5 - Simulated S11 vs measured S11

Fig. 6 shows the simulated gain of the antenna. The gain of $2.42 \mathrm{dBi}$ is obtained. The following $2 \mathrm{D}$ far-field of the antenna is presented in Fig. 7. 


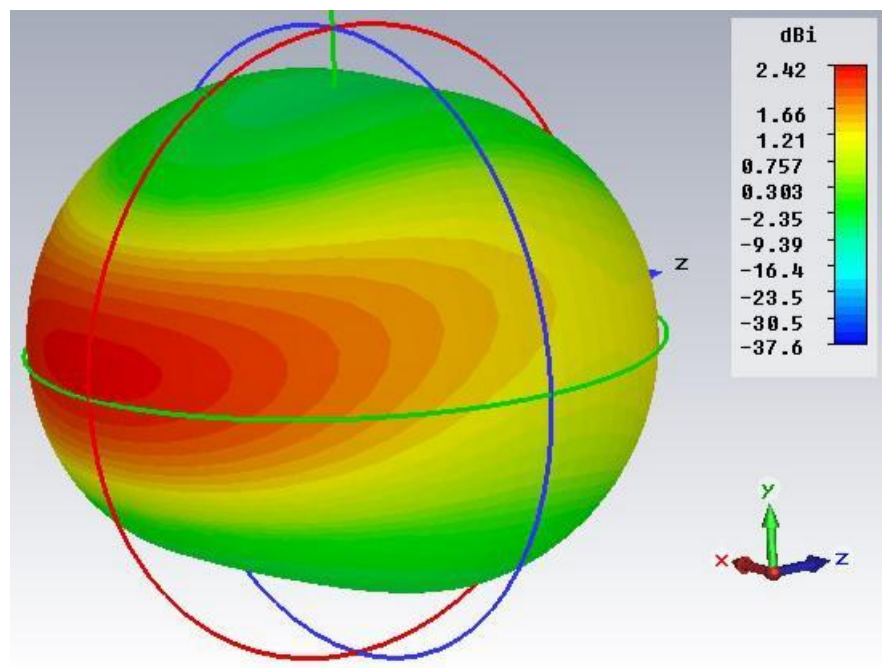

Fig. 6 - The simulated gain of the proposed antenna

Fig. 7 shows the radiation pattern of the antenna between simulation and measurement. The antenna is directed to the organ of the body where the rear position of the antenna is facing the organ, so the highest gain is needed on that side in order the detection process is more accurate. Fig. 7(a) shows the rear position in 180 degrees, while Fig. 7(b) shows the rear position at 0 degrees.

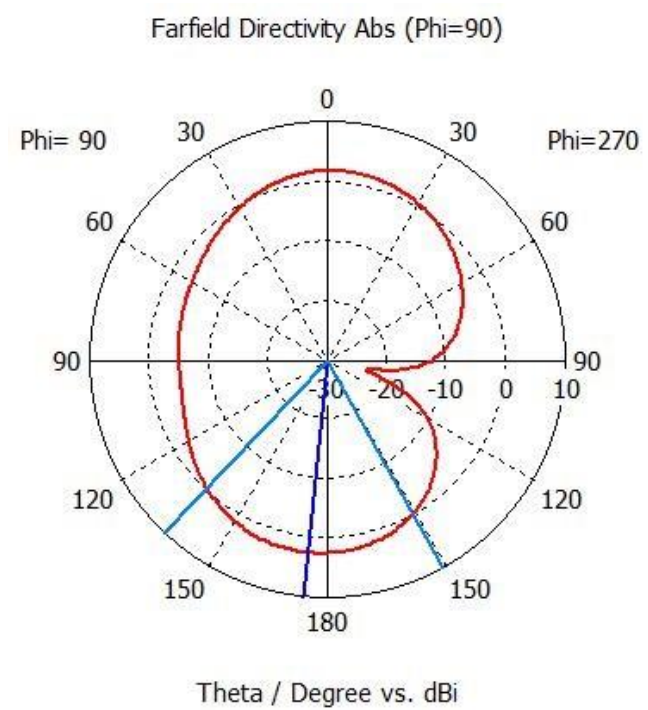

(a)

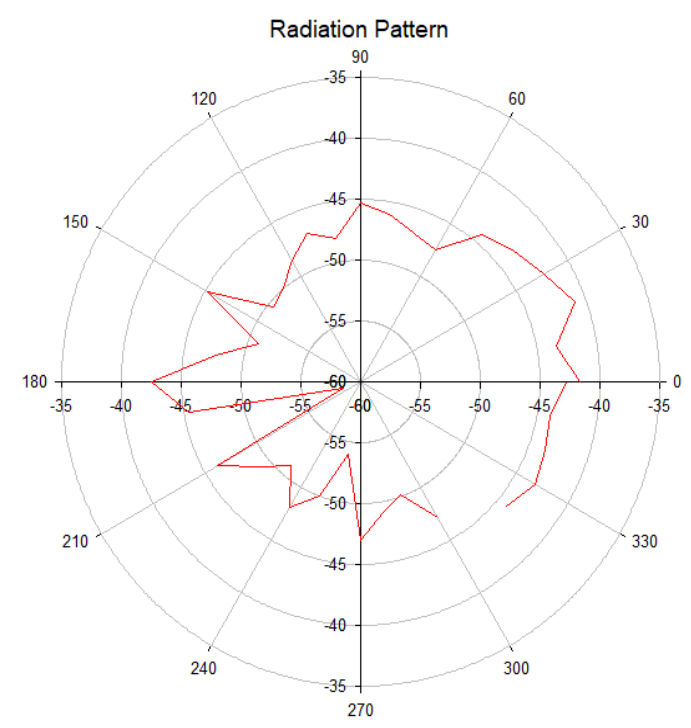

(b)

Fig. 7 - Radiation pattern of the proposed antenna (a) Simulation; (b) Measurement

Fig. 8 shows that the highest current distribution of the antenna is concentrated on the transmission line field. The maximum current distribution of $32.6 \mathrm{~A} / \mathrm{m}$ is achieved. Furthermore, the currents flow toward the patch with decreasing value. 

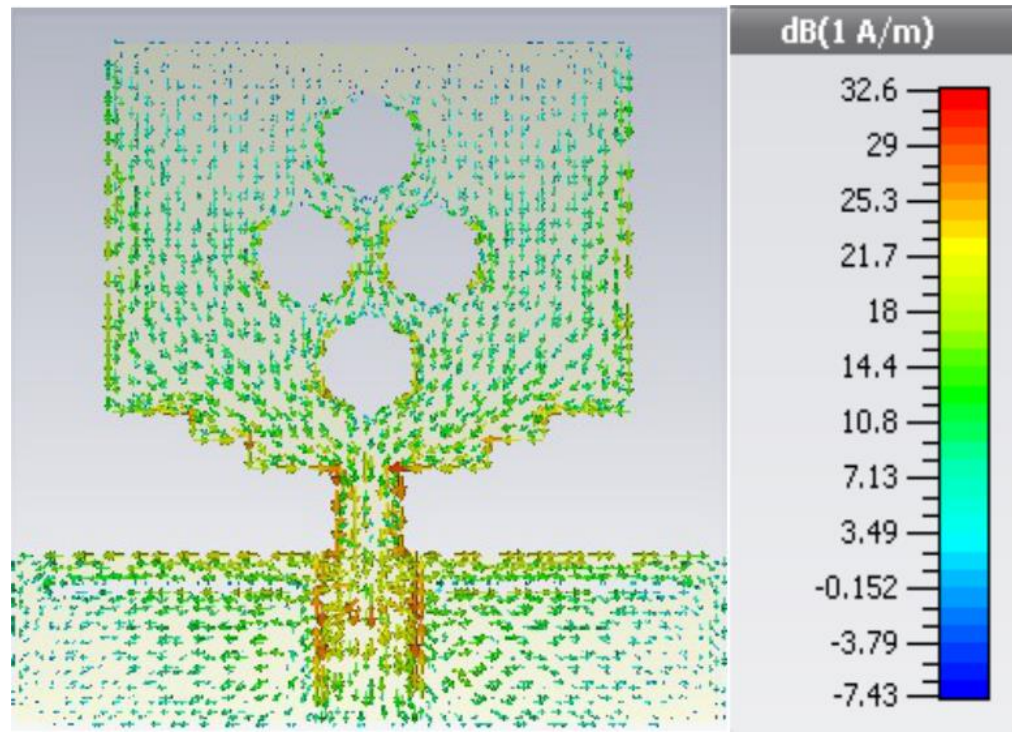

Fig. 8 - Current distribution of the proposed antenna

\section{Comparison with the Existing Research}

The UWB antennas have been studied extensively by many researchers. The research of antenna is applied to biomedical applications. However, The design of the antenna must be in accordance with health standards and convenience in use. Some of the researchers designed the antenna using a hard substrate, such as FR-4 [5][9]. The antenna properties are acquired (i.e., bandwidth, return loss, gain, and radiation pattern) for the best values. So that it can be used for biomedical applications.

Nevertheless, the antennas have a shortcoming that is the substrate. The substrate is so hard that making the detection process sometimes inaccurate. Because the object being examined has different structural shapes. Therefore, other innovations were done by some of the researchers in terms of substrate usage. A substrate model that can be used for biomedical applications is flexible structures. The flexible substrate is believed to increase the accuracy of detecting objects in body organs that have different structures. Here are some of the antenna researchers for biomedical applications that use a flexible substrate shown in Table 3.

Table 3 - Comparison of the antenna properties

\begin{tabular}{|c|c|c|c|c|}
\hline $\begin{array}{l}\text { Reference } \\
\text { No. }\end{array}$ & Substrate & $\begin{array}{l}\text { Bandwidth } \\
(\mathrm{GHz})\end{array}$ & $\begin{array}{l}\text { Dirctivity } \\
(\mathrm{dBi})\end{array}$ & $\begin{array}{l}\text { Radiation } \\
\text { Pattern }\end{array}$ \\
\hline [10] & $\begin{array}{l}\text { Roger } \\
\text { Ultralam } \\
3850\end{array}$ & $\begin{array}{l}110 \mathrm{MHz} \\
\& 600 \\
\mathrm{MHz}\end{array}$ & $\begin{array}{l}1.13 \& \\
3.17\end{array}$ & Bidirectional \\
\hline [11] & Polyester & $4.97-11.73$ & $\begin{array}{l}2.41 \& \\
4.53 \\
\end{array}$ & Bidirectional \\
\hline [12] & Cotton & $1.6-11.2$ & 6.17 & Bidirectional \\
\hline This Work & PET & $2.08-9.96$ & $2.42 \mathrm{dBi}$ & Bidirectional \\
\hline
\end{tabular}

Table 3 shows a comparison of the antennas using a flexible substrate. The authors used Roger ultralam 3850 as a substrate. The simulation results showed the bandwidth of $110 \mathrm{MHz} \& 600 \mathrm{MHz}$ achieved with the directivity of 1.13 $\mathrm{dBi} \& 3.17 \mathrm{dBi}$ at resonance frequencies. Other researchers used Polyester as a substrate. The simulation results showed the bandwidth of $6.76 \mathrm{GHz}$ achieved with the directivity of $2.41 \mathrm{dBi} \& 4.53 \mathrm{dBi}$ at the resonance frequencies. Lastly, the authors also used Cotton as a substrate. The simulation results showed the bandwidth of $9.6 \mathrm{GHz}$ achieved with the directivity of $6.17 \mathrm{dBi}$ at the resonance frequency. All the papers have a bidirectional radiation pattern. Thus, the proposed antenna in this paper also used the flexible substrate for biomedical applications. These results are acceptable and comparable. 


\section{Conclusion}

The proposed stair-hexagonal slot antenna is successfully designed and printed on the PET substrate. The antenna uses a flexible substrate and CPW technique, which can increase the accuracy of object detection. The antenna works well at frequency $5.8 \mathrm{GHz}$ with $7.88 \mathrm{GHz}$ of bandwidth. It can be used for biomedical applications. The simulated gain of the antenna is $2.42 \mathrm{dBi}$ with a bidirectional radiation pattern.

\section{Acknowledgment}

The work is financially supported by the National Agency for Research and Innovation (BRIN), Indonesia, under the INSINAS Program. The authors would also like to thanks LPPM Universitas Riau and the Indonesian Institute of Sciences for their motivation. The authors would like to thanks to the wireless communication center Universiti Teknologi Malaysia for their research collaboration.

\section{References}

[1] di Clemente, F. S., Stephan, R., \& Hein, M. A. (2013, April). Ultra-wideband miniaturised high permittivity-matched antennas for biomedical diagnostic. In 2013 7th European Conference on Antennas and Propagation (EuCAP) (pp. 2896-2899). IEEE

[2] Bray, F., Ferlay, J., Soerjomataram, I., Siegel, R. L., Torre, L. A., \& Jemal, A. (2018). Global cancer statistics 2018: GLOBOCAN estimates of incidence and mortality worldwide for 36 cancers in 185 countries. CA: a cancer journal for clinicians

[3] Kwon, S., \& Lee, S. (2016). Recent advances in microwave imaging for breast cancer detection. International journal of biomedical imaging, 2016

[4] Bassi, M., Caruso, M., Khan, M. S., Bevilacqua, A., Capobianco, A. D., \& Neviani, A. (2013). An integrated microwave imaging radar with planar antennas for breast cancer detection. IEEE Transactions on Microwave Theory and Techniques, 61(5), 21082118

[5] Wasusathien, W., Santalunai, S., Thosdeekoraphat, T., \& Thongsopa, C. (2014). Ultra wideband breast cancer detection by using SAR for Indication of the tumor location. International Journal of Medical, Health, Biomedical, Bioengineering and Pharmaceutical Engineering, 8(7)

[6] Faisal, M. M. A., Uddin, M. J., Ullah, M. W., Kamrul, M. I., Haque, K. M., \& Rahman, E. (2017, November). Comparative analysis of different types of breast cancer cell detection antennas. In 2017 International Conference on Inventive Computing and Informatics (ICICI) (pp. 927-930). IEEE

[7] Rahayu, Y., Rahman, T. A., Ngah, R., \& Hall, P. S. (2008, May). Ultra wideband technology and its applications. In Wireless and Optical Communications Networks, 2008. WOCN'08. 5th IFIP International Conference on (pp. 1-5). IEEE

[8] Katbay, Z., Al Mokdad, S., Sadek, S., Le Roy, M., Lababidi, R., \& Pérennec, A. (2017, September). A UWB antenna in direct breast contact for cancer detection. In 2017 Sensors Networks Smart and Emerging Technologies (SENSET) (pp. 1-4). IEEE

[9] Subramanian, S., Sundarambal, B., \& Nirmal, D. (2018). Investigation on Simulation-Based Specific Absorption Rate in UltraWideband Antenna for Breast Cancer Detection. IEEE Sensors Journal, 18(24), 10002-10009

[10] Kadry, M., El Atrash, M., \& Abdalla, M. A. (2018, July). Design of an Ultra-thin Compact Flexible Dual-Band Antenna for Wearable Applications. In 2018 IEEE International Symposium on Antennas and Propagation \& USNC/URSI National Radio Science Meeting (pp. 1949-1950). IEEE

[11] Wang, F., Arslan, T., \& Wang, G. (2017, October). Breast cancer detection with microwave imaging system using wearable conformal antenna arrays. In 2017 IEEE International Conference on Imaging Systems and Techniques (IST) (pp. 1-6). IEEE

[12] Alsharif, F., \& Kurnaz, C. (2018, July). Wearable Microstrip Patch Ultra Wide Band Antenna for Breast Cancer Detection. In 2018 41st International Conference on Telecommunications and Signal Processing (TSP) (pp. 1-5). IEEE

[13] Garg, R., Bhartia, P., Bahl, I. J., \& Ittipiboon, A. (2001). Microstrip antenna design handbook. Artech house

[14] Alibakhshi-Kenari, M., Naser-Moghadasi, M., Sadeghzadeh, R. A., Virdee, B. S., \& Limiti, E. (2016). Bandwidth extension of planar antennas using embedded slits for reliable multiband RF communications. AEU-International Journal of Electronics and Communications, 70(7), 910-919 